\title{
Migratory Phenomenons, Bioethics and Vaccinations
}

\author{
Giulio Tarro1,2 \\ ${ }^{1}$ Department of Biology, Center for Biotechnology, Sbarro Institute for Cancer Research and Molecular \\ Medicine, Temple University, Philadelphia, PA, USA \\ ${ }^{2}$ Committee on Biotechnologies and Virus Sphere, World Academy of Biomedical Technologies, UNESCO, \\ Paris, France \\ Email: gitarro@tin.it, giuliotarro@gmail.com
}

Received 1 September 2015; accepted 25 September 2015; published 28 September 2015

Copyright (C 2015 by author and Scientific Research Publishing Inc.

This work is licensed under the Creative Commons Attribution International License (CC BY).

http://creativecommons.org/licenses/by/4.0/

c) (i) Open Access

\begin{abstract}
The evolution of the HIV following migratory phenomenons is reported in an interesting study carried on for 15 years. Polymerase Chain Reaction (PCR) is a technique in molecular biology that amplifies a specific region of deoxyribonucleic acid (DNA), and has been useful in the molecular characterization of viruses as poxviruses. In many countries, such as Italy, the vaccinations are included in the so-called sanitary obliged not coercive treatments. This means that the obligation is anticipated for all the subjects belonging to one determined range of age to undergo some vaccinations, but these are not subministrated by force in case of refusal. The legitimacy of the obligatory vaccination against the smallpox, with all the risks connected, stated that such "risks were too much reduced to be seriously taken in consideration for the benefits produced on the collectivity". To ratify this obligation brings, however, to the reimbursement of the incidental damages produced by the vaccination. Nevertheless, mass vaccination against smallpox continues to be a leading initiative in western countries to guard against bioterrorist attack.
\end{abstract}

\section{Keywords}

HIV, Poxviruses, Vaccines, Bioterrorism

\section{Introduction}

The following paper has three sections: the first one is concerning with the phenomenon of immigrants that represent just now an up to date evidence of the lack of an uniform health policy of Europe for such people. The observation of increased incidence of sexually transmitted diseases (STD) and HIV/AIDS among the foreigners 
in Italy resulted by the application mostly of PCR that has represented therefore the main topic of the second section. According to the history of the studies on PCR, this technique was very important for rapid identification and differentation of orthopoxviruses. The main component of this viral family for human pathology was the agent of the smallpox, the first disease to be eradicated by a vaccine. This virus became "fashionable" for the threat of bioterrorism that then has been the reason to be dealt with in the third section.

\section{The Evolution of the HIV Following Migratory Phenomenons}

I like to report an interesting study carried on for 15 years.

From the first of January 1985 to September $30^{\text {th }} 1999$, at the service of Preventive Medicine of the Migrations, of the Tourism and of Tropical Dermatology of the Institute S. Gallicano (IRCS) of Rome, by the first visit have been submitted 34.415 people immigrated irregularly and clandestine, of which 12.970 (37.68\%) females and 21.445 (62.32\%) males; $3.440(10 \%)$ had an inclusive age between 0 and 12 years. The diagnosis of STD has represented the $12 \%$ of the total diagnosis effected by the Service, with a trend in progressive increase, particularly in comparison to the first half of the years 80 . The $78 \%$ of the cases of sexually transmitted disease (STD) have been observed in men, with a 24-year-old average age, for $94 \%$ heterosexual. In the first 9 months of the " 99 have been visited 5.122 immigrated people, clandestine and irregalor". Among these 662 people have been selected without fixed residence (SFD) (263 women and 399 men) divided as it followes: 466 Italians and 196 foreigners; $20.8 \%$ were less than 25 years old, $34.8 \%$ on inclusive age between 25 and 36 years, $29.9 \%$ between 37 and 50 years and $14.5 \%$ greater than 50 years. To the selected population the execution of the test has been proposed for the search of the anti-HIV antibodies (Ab) [1] and over $80 \%$, have expressed their own consent.

The percentage of subjects with HIV/AIDS infection [2] in the Italian population (SFD) was $2.8 \%$, while in the foreigner population was $10.1 \%$.

The positive foreign patients originated for 36.0\% from Africa, for 18.0\% from Latin America, for 22.0\% from Asia and for $24.0 \%$ from East Europe. The last data (June 1999) from the AIDS Operational Center of the Superior Institute of Health (ISS) underline, with the second semester of 1995 a rapid and constant diminution of the AIDS incidence; such diminution however seems to concern only the cases of AIDS related to people of Italian nationality. In fact the percentage distribution of the cumulative cases of AIDS for nationality in our Country underlines in the time a continuous increase of the AIDS cases among foreign citizens: we passed in fact from the 3.0\% of AIDS cases in foreign citizens in the inclusive period between 1982 and 1993 to $10.0 \%$ in the first semester of 1999. More than half of them (5.07\%) originate from Africa, 1.58\% from the South America, $0.79 \%$ from East Europe $0.63 \%$ from Asia, $0.32 \%$ from North America and for $1.58 \%$ it was not possible to go up to the geogrophical area of origin.

Even if the data concern to a limited number of patients and one cannot correctly compare with the national ones that refer cases only of AIDS, the increased incidence of STD and HIV/AIDS among the foreigners, would confirm the hypothesis, already observed for other pathologies, that a greater number of foreign people decides to migrate for taking care of themselves. If further studies in other European countries would confirm this "trend", taking in consideration the elevated cost of the actual antiretroviral therapies, the next politics of Public Health in Europe have to keep in mind of the changed perspectives of prevention, diagnosis and therapy. More direct investigations reported with following optional questionnaires fortify this intuition.

\section{Polymerase Chain Reaction and Poxviruses}

Polymerase Chain Reaction (PCR), is a technique in molecular biology that amplifies a specific region of deoxyribonucleic acid (DNA). This specific region of DNA can be rapidly cloned, or duplicated, to produce multiple DNA copies. This enables scientists to make billions of copies of a DNA molecule in a very short time. PCR allows scientists to adequately test the DNA to detect: DNA sequences, diagnose genetic diseases, carry out DNA fingerprinting \{identify individuals\}, bacteria [3] or viruses, and research human evolution [2].

PCR goes through a 3-phase process, which proceeds in a series of cycles, or rounds. The DNA fragment is duplicated after one successive cycle. Thus, more than one billion copies of a single DNA fragment can be made in just a few hours. The technique of PCR is very straightforward that scientists with little training in molecular biology can use it. The use of PCR has a very high probability achievement. The supplies necessary for carrying out PCR are available in a kit form manufactured ad hoc. This kit is used in such varied settings as crime labor- 
atories and clinical diagnostic laboratories.

Rapid identification and differentiation of orthopoxviruses by PCR were achieved with primers based on genome sequences encoding the hemagglutinin (HA) protein, an infected-cell membrane antigen that distinguishes orthopoxviruses from other poxvirus genera.

The initial identification step used a primer: pair of consensus sequences for amplifying an HA DNA fragment from the three known North American orthopoxviruses (raccoonpox, skunkpox, and volepox viruses), and a second pair for amplifying virtually the entire HA open reading frame of the Eurasian-African orthopoxviruses (variola, vaccinia, cowpox, monkeypox, camelpox, ectromelia, and gerbilpox viruses). Residual digest electropherograms of the amplified DNAs of the former subgroup provided species differentiation, and Taql digests differentiated the Eurasian-African orthopoxviruses, including vaccinia virus from the vaccinia virus subspecies buffalopox virus. Endonuclease Hhal digest patterns distinguished smallpox variola major viruses from alastrim variola minor viruses.

For the Eurasian-African orthopoxviruses, a confirmatory step that used a set of higher-sequence-homology primers was developed to provide sensitivity to discern individual virus HA DNAs from cross-contaminated orthopoxvirus DNA samples; Taql and Hhal digestions of the individual amplified HA DNAs confirmed virus identity.

Finally, a set of primers and modified PCR conditions were developed on the basis of base sequence differences within the HA genes of the 10 species, which enabled production of a single DNA fragment of a particular size that indicated the specific species.

\section{The Obligation of the Vaccinations and the Threat of Missing Therapies}

In many countries, such as Italy, the vaccinations [4] are included in the so-called sanitary obliged not coercive treatments. This means that the obligation is anticipated for all the subjects belonging to one determined range of age to undergo some vaccinations, but these are not subministrated by force in case of refusal. Not all the countries choose this road to make sure that the objective of the diffusion of the vaccination is reached, and they resort to campaign of sanitary education (it is so, traditionally, in Great Britain) or to forms of indirect coercion, as the prohibition of access to the scholastic services or, in general, to the services disbursed with public financing (it is the case of the United States, where however some vaccinations are made also obligatory).

In the United States the Federal Supreme Court with a sentence destined to make reference for the rest of the world didn't show any doubts to resolve the controversy promoted, by those people who contested, in name of the individual liberty and the right of the single to the self-determination, the legitimacy of the imposition of the obligatory vaccination against the smallpox [2], with all the risks connected. The Court stated that such "risks were too much reduced to be seriously taken in consideration for the benefits produced on the collectivity". To ratify this obligation brings, however, to the reimbursement of the incidental damages produced by the vaccination. The matter was resoundingly set in 1968 when a Court of Federal Appeal of the United States condemned for the first time the producer of an antipolio vaccine to indemnify a subject obligatorily vaccinated that the polio had contracted, since he was not duly warned on any consequential risk with the assumption of the vaccine. This sentence has had disruptive effects because it has brought the exponential growth of the applications of reimbursement. In 1982, a campaign of compelled vaccination against a particularly dangerous influenza has involved over 4000 judicial controversies, with a question of reimbursement towards the producers of vaccines equal to 3 million dollars. This has brought the impossibility to retrieve a number of producers of medicines that consented to make vaccines, because the insurance companies refused to protect the manufacturing factories asking for exorbitant costs. In 1985, of the ten producers of vaccines on the market fifteen years earlier only three were left. Also the scientific research in the sector resulted in clean diminution, the investments having been addressed to other fields despite the market of the vaccinations in the United States is worth, according to official reports, over 500 million dollars a year. The legislative answers to the danger sanitary and public provoked by this situation have been a difficult return to the past, and therefore to exclude reimbursements of damages, or to set the obligation of reimbursement to the state (anticipated for the first time in a 1976 law - the Swine Flu Act) [5]. A road, this one, followed already by many European countries (from 1963 Germany and from 1964 France) such as Italy, is added, also with the law 210 of February 25, 1992.

And the research activities of the great pharmaceutical companies have discovered the needs of vast areas of the planet; it is enough to think that of the 1.233 new products put into the market between 1975 and 1997, only 13 have been approved as specific treatments for tropical pathologies, including some most lethal illnesses of the 
world. At the same time, legal matters make often difficult the access to the medical remedies, as it happens with their patents that prevent the countries of the third world, seriously struck by the virus of the AIDS, the local production of the anti-retroviral drugs. Also when the products are available and not subject to the limitations imposed by the licences, the costs keep on constituting the principal obstacle. According to the WHO, one third of the human population doesn't have regular access to therapies of fundamental importance, which would warrant the care of the most part of the infections and the chronic illnesses that torment the world. From the half of the 80 years, this slice of population has nearly been unchanged, and it reaches $50 \%$ in the poorest countries, despite an individual cost esteemed in only two dollars. In 1998, a child of four has not received the six principal treatments of vaccine prophylaxis for polio, diphtheria, pertussis, tetanus, measles and tuberculosis. And the slaughter goes on.

\section{Acknowledgements}

Financial support Foundation T. \& L. de Beaumont Bonelli for cancer research, Naples Italy www.fondazione-bonelli.org, fondazionebonelli@gmail.com.

\section{Conflict of Interest}

The author declares no conflict of interests.

\section{References}

[1] Tarro, G. (2014) New Development on Tumor Associated Antigen with Specific Target toward Lung Cancer. International Journal of Research in Medical and Health Sciences, 3, 2307-2083.

[2] Tarro, G. (2013) Origin of the Viruses and Their Evolutionary History. Advance Studies in Biology, 5, 423-430.

[3] Tarro, G. and Altucci, P. (2014) Mycoplasmas and Mycoplasmosis: 20 Years Ago. Atti dell'Accademia Lancisiana Simposio Il Policlinico, 121, 229-245.

[4] Tarro, G. (2014) Viral Latency, Molecular Pathogenesis and Malignancy. Global Journal of Science Frontier Research: C Biological Science, 14, 2249-4626.

[5] Tarro, G. and Esposito, C. (2011) Emerging Viral Agents at Risk in Global Health Approaches to Early Diagnosis and Prompt Therapy. International Conference on Bioinformatics and Computational Biology (BIOCOM'11), Las Vegas, Nevada, 18-21 July 2011. 